\title{
A Note on the Zeros of One Form of Composite Polynomials
}

\author{
Dragomir M. Simeunović
}

Abstract. In this paper we consider one form of composite polynomials. Several relations concerning their zeros are obtained.

Let $P(z)$ be a polynomial

$$
P(z)=a_{0}+a_{1} z+a_{2} z^{2}+\cdots+a_{n} z^{n}, \quad a_{n} \neq 0,
$$

whose zeros $z_{1}, z_{2}, \ldots, z_{n}$ are arranged so that

$$
\left|z_{1}\right| \leq\left|z_{2}\right| \leq \ldots \leq\left|z_{n}\right|
$$

and a polynomial

$$
Q(z)=P(z)-c_{k} z^{k}
$$

where $k$ is a fixed integer $(1 \leq k \leq n), c_{k}$ is an arbitarary constant and it holds that $c_{n} \neq a_{n}$.

Let $u_{1}, u_{2}, \ldots, u_{n}$ be the zeros of the polynomial $Q(z)$ arranged so that

$$
\left|u_{1}\right| \leq\left|u_{2}\right| \leq \ldots \leq\left|u_{n}\right|
$$

Then:

$$
\begin{aligned}
& \left|P\left(u_{1}\right)\right| \leq\left|P\left(u_{2}\right)\right| \leq \ldots \leq\left|P\left(u_{n}\right)\right| . \\
& \left|Q\left(z_{1}\right)\right| \leq\left|Q\left(z_{2}\right)\right| \leq \ldots \leq\left|Q\left(z_{n}\right)\right| .
\end{aligned}
$$

(C) Besides every zero $u_{i}$ of the polynomial $Q(z)$ there exists at least one zero $z_{j}$ of the polynomial $P(z)$ such that

$$
\left|z_{j}-u_{i}\right| \leq\left(\left|\frac{c_{k}}{a_{n}}\right|\left|u_{i}\right|^{k}\right)^{\frac{1}{n}} \leq\left(\left|\frac{c_{k}}{a_{n}}\right| M_{q}^{k}\right)^{\frac{1}{n}},
$$

where $u_{q}$ is the upper bound of the moduli of zeros of the polynomial $Q(z)$. (D) Besides every zero $z_{i}$ of the polynomial $P(z)$ there exists at least one zero $u_{s}$ of the polynomial $Q(z)$ such that

$$
\left|u_{s}-z_{i}\right| \leq\left(\left|\frac{c_{k}}{a_{n}}\right|\left|z_{i}\right|^{k}\right)^{\frac{1}{n}} \leq\left(\left|\frac{c_{k}}{a_{n}}\right| M_{p}^{k}\right)^{\frac{1}{n}},
$$

2000 Mathematics Subject Classification. Primary: 12D10.

Key words and phrases. Roots of algebraic equations, upper bounds for roots moduli. 
where $u_{p}$ is the upper bound of the moduli of zeros of the polynomial $P(z)$.

Before we give proofs of (A)-(D), we represent polynomials $P(z)$ and $Q(z)$ in the following form

$$
\begin{aligned}
& P(z)=a_{n}\left(z-z_{1}\right)\left(z-z_{2}\right) \cdots\left(z-z_{n}\right), \\
& Q(z)=a_{n}\left(z-u_{1}\right)\left(z-u_{2}\right) \cdots\left(z-u_{n}\right) .
\end{aligned}
$$

Proof (A). From the equation (7), it follows that

$$
P\left(u_{i}\right)=c_{k} u_{i}^{k}, i=1,2, \ldots, n,
$$

that is

$$
\left|P\left(u_{i}\right)\right|=\left|c_{k}\right|\left|u_{i}\right|^{k}, i=1,2, \ldots, n,
$$

wherefrom, because of (4), we conclude that (A) holds.

Proof (B). From the equation (3), it follows that

$$
Q\left(z_{i}\right)=-c_{k} z_{i}^{k}, i=1,2, \ldots, n,
$$

that is

$$
\left|Q\left(z_{i}\right)\right|=\left|c_{k}\right|\left|z_{i}\right|^{k}, i=1,2, \ldots, n,
$$

wherefrom, because of (2), we conclude that (A) holds.

Proof (C). The equation (9), according to (7), is reduced to the equation

$$
\left|a_{n}\right|\left|u_{i}-z_{1}\right|\left|u_{i}-z_{2}\right| \cdots\left|u_{i}-z_{n}\right|=\left|c_{k}\right|\left|u_{i}\right|^{k}, i=1,2, \ldots, n .
$$

Let $z_{j}$ be a zero of the polynomial $P(z)$ that is closest to the zero $u_{i}$ of the polynomial $Q(z)$. Then, from (11) we get

$$
\left|a_{n}\right|\left|u_{i}-z_{j}\right|^{n} \leq\left|c_{k}\right|\left|u_{i}\right|^{k}
$$

wherefrom (C) follows.

Proof (D). The equation (10), according to (8), is reduced to the eqaution

$$
\left|a_{n}\right|\left|z_{i}-u_{1}\right|\left|z_{i}-u_{2}\right| \cdots\left|z_{i}-u_{n}\right|=\left|c_{k}\right|\left|z_{i}\right|^{k}, i=1,2, \ldots, n .
$$

Let $u_{s}$ be a zero of the polynomial $Q(z)$ that is closest to the zero $z_{i}$ of the polynomial $P(z)$. Then, from (12) we get

$$
\left|a_{n}\right|\left|z_{i}-u_{s}\right|^{n} \leq\left|c_{k}\right|\left|z_{i}\right|^{k}
$$

wherefrom (D) follows.

The case where $k=0$ and $c_{0}=c$ is given in [1, p. 80]. The case where $k=n$ and $c_{n}=a_{n}$ is given in [1. p.80], and also in [2] and [3]. 


\section{REFERENCES}

[1] M. Marden, Geometry of Polynomials, Amer. Math. Soc. Providence, R.I. 2005.

[2] G. Szegö, Bemerkungen zu einem Satz von J. H. Grace über die Wurzeln algebraischer Gleichungen, Math. Zeit., Vol. 13, 1922, 28-55.

[3] D. M. Simeunović, Sur le cercle qui contient au moins un zéro d'un polynôme et les questions qui s'y rattachent, Mat. Vesnik, Vol. 5, No. 20, 1968, 339-342.

Dragomir M. Simeunović Mike Alasa 8

11000 BELGRADE

SERBia 
